\title{
Uncovering unsuspected advanced liver fibrosis in patients referred to alcohol nurse specialists using the ELF test
}

Freya Rhodes ${ }^{1,6+}$, Sara Cococcia ${ }^{1,2,6+}$, Jasmina Panovska-Griffiths ${ }^{3,4}$, Sudeep Tanwar ${ }^{1,5}$, Rachel H. Westbrook ${ }^{1,6}$, Alison Rodger ${ }^{4}$ and William M. Rosenberg ${ }^{1 *}$ (D)

\begin{abstract}
Background and aims: Alcohol use disorders (AUD) cause 7.2\% of UK hospital admissions/year. Most are not managed by hepatologists and liver disease may be missed. We used the Enhanced Liver Fibrosis (ELF) test to investigate prevalence and associations of occult advanced liver fibrosis in AUD patients not known to have liver fibrosis.

Methods: Liver fibrosis was assessed using ELF in prospective patients referred to the Royal Free Hospital Alcohol Specialist Nurse (November 2018-December 2019). Known cases of liver disease were excluded. Patient demographics, blood tests, imaging data and alcohol histories recorded. Advanced fibrosis was categorised as ELF $\geq 10.5$.

Results: The study included 99 patients (69\% male, mean age $53.1 \pm 14.4)$ with median alcohol intake 140 units/ week (IQR 80.9-280), and a mean duration of harmful drinking of 15 years (IQR 10-27.5). The commonest reason for admission was symptomatic alcohol withdrawal (36\%). The median ELF score was 9.62, range 6.87-13.78. An ELF score $\geq 10.5$ was recorded in 28/99 (29\%) patients, of whom $28.6 \%$ had normal liver tests. Within previous 5 -years, $76 \%$ had attended A\&E without assessment of liver disease. The ELF score was not associated with recent alcohol intake $(p=0.081)$, or inflammation $(p=0.574)$.

Conclusion: Over a quarter of patients with AUD had previously undetected advanced liver fibrosis assessed by ELF testing. ELF was not associated with liver inflammation or recent alcohol intake. The majority had recent missed opportunities for investigating liver disease. We recommend clinicians use non-invasive tests to assess liver fibrosis in patients admitted to hospital with AUD.
\end{abstract}

Keywords: Liver cirrhosis, Liver diseases, Alcoholic, Non-invasive test, Alcohol use disorder, Enhanced liver fibrosis test

*Correspondence: w.rosenberg@ucl.ac.uk

${ }^{\dagger}$ Freya Rhodes and Sara Cococcia are joint 1st authors

${ }^{1}$ Institute for Liver and Digestive Health, Division of Medicine, University

College London, Royal Free Campus, Rowland Hill Street, Hampstead,

London NW3 2PF, UK

Full list of author information is available at the end of the article

\section{Highlights}

- $1 / 4$ patients in hospital with AUD had ELF scores $\geq 10.5$ indicative of advanced fibrosis.

- $3 / 4$ patients with AUD had attended A\&E within 5yrs without liver fibrosis assessment

- ELF score was not associated with inflammation (AST or ALT)

- ELF score was not associated with amount of alcohol consumed (Units/Week) 
- $28 \%$ of those with advanced fibrosis (ELF $\geq 10.5)$ had normal liver function tests

\section{Background}

One in five people in the UK drink alcohol at hazardous or harmful levels [1]. While alcohol causes a wide array of health and social harms, the greatest morbidity and mortality are associated with alcohol-related liver disease (ArLD) with mortality rates increasing 400\% since 1970 [2].

Hospital admissions related to alcohol are rising annually, with 350,000 alcohol related admissions per year in 2019, (an increase of $20 \%$ in a decade) [3] and with a cost to the NHS of $£ 3.5$ billion per year [4]. This is likely to be due to a shift in drinking behaviours from low-strength beer in pubs to home consumption of higher strength beer, wine and spirits sold in supermarkets. In addition, alcohol is now $64 \%$ more affordable than it used to be 30 years ago [1].

While a proportion of people admitted to hospital with harms arising from their drinking behaviour are recognised to have liver fibrosis and are managed by liver specialists, many are managed by a wide range of doctors and their liver disease may be missed, even if their alcohol use disorder (AUD) is recognised.

Moreover, it is estimated that up to $75 \%$ of people with Chronic Liver Disease (CLD) first present to healthcare when their liver disease is advanced often with decompensated cirrhosis, when it is too late for behaviour change or interventions to avert poor outcomes $[1,5,6]$.

Part of the reason for this is because cirrhosis is often asymptomatic, and it is not reliably detected using routine liver function blood tests (LFTs) or ultrasound [7]. The last two decades have witnessed the development and validation of a number of non-invasive tests for liver fibrosis that are increasingly used in clinical practice. These tests create the possibility to detect advanced liver fibrosis in at-risk patients with AUD, in order to refer them appropriately to hepatology services for regular follow up to avert, or detect and treat complications of portal hypertension including oesophageal varices $[8,9]$ and ascites [10], and screening for liver cancers [11, 12], as well as alcohol counselling from hospital or community services as appropriate.

Currently non-invasive testing for liver fibrosis with the Enhanced Liver Fibrosis test (ELF) is widely used for Non-Alcoholic-Fatty Liver Disease (NAFLD) to determine which patients with fatty liver have advanced fibrosis and warrant referral to hepatology, versus those with low ELF scores who can remain in primary care [13]. However, although evidence-based [14] and recommended by BSG guidance, [7] this approach is not yet in widespread use in the NHS for people with AUD.

We aimed to investigate the prevalence of advanced liver fibrosis using the ELF test employing the literaturebased ELF cut-off of 10.5 [14], in patients recognised to have AUD but not recognised as having liver disease. In addition, we aimed to examine the relationship between demographic factors (including age, Body Mass Index (BMI), deprivation score, baseline LFTs and the ELF score.

\section{Methods}

\section{Study design}

This was a prospective service evaluation conducted at the Royal Free Hospital from November 2018 to December 2019.

\section{Patient population}

Consecutive referrals to the Royal Free Hampstead alcohol specialist nurse (ASN) were included if aged $\geq 18$ and excluded if already under the care of a hepatologist, if they had a known chronic liver condition, a diagnosis of alcoholic hepatitis, or acute liver injury secondary to a cause other than alcohol. Out of 100 consecutive referrals to the ASN 98 were in-patients or emergency department attendees. The vast majority of out-patient referrals were ineligible for inclusion because they had known ArLD, leaving 2/100 eligible out-patient referrals. One patient was excluded from the analysis as they were found to have an ALT of 1,023 following a pregabalin overdose, reducing the sample size from 100 to 99 .

\section{Clinical data}

Data extracted from patients' electronic medical records included patient demographics, reason for presentation to hospital, BMI, alcohol intake (detailed in next section), postcode to enable deprivation score calculation, results of any imaging or fibrosis tests performed within 6 months of referral to ASN, blood test results to enable calculation of FIB4, AST:ALT ratio and APRI [14], and number of hospital presentations within the last five years.

Data were anonymised and entered into a password protected spreadsheet held on a secure NHS computer.

\section{Alcohol data}

Current alcohol consumption was recorded in units per week $(\mathrm{U} / \mathrm{w})$, and duration of 'excess alcohol consumption' in years. This was obtained from patients' selfreported consumption extracted from free text in clinical notes. AUDIT scores were not available. It was noted if the patient had been actively drinking up to the point of presentation to hospital. 


\section{ELF score}

An ELF test was performed on consecutive eligible patients referred to the ASN. Serum was extracted from $5 \mathrm{~mL}$ blood per patient which was analysed at the Central ELF laboratory (iQur Limited, London). The samples were analysed for HA, PIINP and TIMP1 levels using the proprietary assays developed by Siemens Healthineers Inc (Tarrytown, New York, USA) for the ELF test, on a Siemens ADVIA centaur ${ }^{\circledR}$ immunoassay system. ELF Scores were calculated from test results using the manufacturer's published algorithm. An ELF threshold of 10.5 was pre-selected for detection of advanced fibrosis based on recommendations by Thiele et al. [14].

\section{Outcomes}

The primary outcome was the proportion of patients referred to the ASN at the Royal Free Hospital who had previously undetected advanced fibrosis as determined by an ELF score of $\geq 10.5$ [14]. Secondary outcomes investigated potential risk factors for advanced fibrosis including alcohol consumption, BMI, age, sex, deprivation score and smoking status. In addition, missed opportunities for diagnosis of liver disease were assessed by counting the number of attendances to hospital within the previous five years without assessment for liver fibrosis.

\section{Follow up}

Patients with ELF scores $\geq 10.5$ were sent a letter inviting them to attend a hepatology outpatient clinic to see a hepatologist with an interest in ArLD, and to have a FibroScan. Blood samples were also taken to screen for viral, immunological and metabolic causes of liver disease in accordance with current protocols if these tests had been omitted during their hospital admission.

\section{Sample size}

As this is an exploratory investigation, following statistical advice we accepted a precision of estimate at 0.1 which would generate a sample size of between 62 and 89 using literature-based estimates of prevalence of advanced fibrosis. A post-hoc sample size calculation for 0.29 prevalence of advanced fibrosis, using a precision of estimate at 0.1 and $0.95 \%$ CI results in a minimum required sample size of $n=80$.

\section{Statistical analysis}

Demographic information was described using frequencies and percentages for categorical variables. Continuous data were described using means and SD or medians and IQR, depending on the normality of the data.
For the comparison of categorical variables, ChiSquared or, if sample size was less than 5, Fisher's exact test was used as a conventional test, and for the continuous data the Mann Whitney $U$ (for non-parametric data) or Student's $t$ test for normally distributed data.

Alcohol 'units per week' was analysed both as continuous data, and in quartiles. After univariate analyses, to determine the variables associated with the presence of advanced fibrosis with the most significance, a multiple binary logistic regression analysis model was used, using the literature-based ELF threshold of 10.5 [14] and multiple linear regression was used for continuous ELF scores. Variables were selected if they were established in the literature as risk factors for liver fibrosis, and if they had $p$ values less than 0.25 in univariate analyses (either against $\mathrm{ELF}</ \geq 10.5$ or as a continuous variable). All $p$ values were 2 -sided and they were considered significant if $p<0.05$. All data were analysed using SPSS software (Version 25.0. Armonk, NY: IBM Corp).

\section{Results \\ Study demographics}

The analysis included 99 patients (69\% male) with a mean age of 53.1 years (SD 14.4) (Table 1). Average BMI was $26.52 \mathrm{~kg} / \mathrm{m}^{2}$ (SD 5.94) and $84 \%$ were current or past smokers. Alcohol intake was high with a median consumption of $140 \mathrm{U} / \mathrm{w}$ (80.9-280), and in this cohort men and women drank similar amounts $(p=0.73)$. The two patients seen in the ASN outpatient clinic had not been drinking alcohol within the past 3 months, and one inpatient had stopped drinking three weeks prior to hospital admission. All of the other 96/99 patients were drinking alcohol up to the point of presentation to hospital. The median duration of alcohol consumption was 15 years (IQR 10-27.5). This cohort of patients were from deprived areas, with $69 \%$ of them positioned within the lowest 4 deprivation deciles.

\section{Reasons for presentation to healthcare}

The vast majority $(n=97 / 99,98 \%)$ of patients were seen as inpatients or in the emergency department. The most common reason for presentation to hospital was symptomatic alcohol-withdrawal (36.4\%) including seizures, followed by injuries from falling over (13.1\%) and mental health presentations $(11.1 \%)$ including overdose. The vast majority $(73.7 \%)$ were under the care of a general medical team (Fig. 1a, b). In the preceding 5 years $76 \%$ of the patients had attended hospital without being diagnosed as having ArLD (aside from the current visit), with median number of hospital attendances being 4 (IQR 2-9). 
Table 1 Comparison of clinical characteristics of patients with and without advanced fibrosis (as determined by ELF score of $\geq 10.5$ )

\begin{tabular}{|c|c|c|c|c|}
\hline Patient characteristics & Overall $(n=99)$ & $\begin{array}{l}\text { Advanced fibrosis } \\
E L F \geq 10.5\end{array}$ & $\begin{array}{l}\text { Non-advanced fibrosis } \\
E L F<10.5\end{array}$ & $p$ value \\
\hline Age mean sd & $53.11 \pm 14.37$ & $55.7 \pm 12.6$ & $52.1 \pm 15$ & 0.266 \\
\hline Male sex n (\%) & 68/99 (69) & 19/28 (68) & 49/71 (69) & 0.911 \\
\hline BMI mean sd & $26.52 \pm 5.94$ & $26.4 \pm 5.7$ & $26.6 \pm 6.1$ & 0.903 \\
\hline T2DM diagnosis (\%) & 10/99 (10.1\%) & $4 / 28(14.3 \%)$ & $6 / 71(8.5 \%)$ & 0.46 \\
\hline \multicolumn{5}{|l|}{ Smoking status n (\%) } \\
\hline Non-smoker & $15(16)$ & $6(21)$ & $9(13)$ & 0.35 \\
\hline Smoker & $69(73.4)$ & $16(57)$ & $53(75)$ & 0.1 \\
\hline Ex-smoker & $10(10.6)$ & $5(18)$ & $5(7)$ & 0.14 \\
\hline Unknown & $5(5)$ & $1(4)$ & $4(6)$ & \\
\hline Ongoing active drinking $n(\%)^{\mathrm{a}}$ & 96/99 (97) & $26 / 28(93)$ & 70/71 (99) & 0.19 \\
\hline Current alcohol intake $\mathrm{U} / \mathrm{W}$, median (IQR) & $140(80.9-280)$ & $112(70-210)$ & $150(105-280)$ & 0.031 \\
\hline Years of harmful drinking median (IQR) & $15(10-27.5)$ & $20(10-28)$ & $15(7.5-28)$ & 0.357 \\
\hline \multicolumn{5}{|l|}{ Signs of CLD on exam } \\
\hline Yes n (\%) & $4(4 \%)$ & $4(14.3)$ & $0(0 \%)$ & 0.005 \\
\hline No n (\%) & 95 (96\%) & $24(85.7)$ & $71(100 \%)$ & \\
\hline \multicolumn{5}{|l|}{ Abnormal LFTs at referral ${ }^{b}$} \\
\hline Yes & $63(66.3 \%)$ & $18(64.3)$ & $45(63.4 \%)$ & 0.712 \\
\hline No & $32(33.7 \%)$ & $8(28.6)$ & $24(33.8 \%)$ & \\
\hline$N=$ & 95 & 26 & 69 & \\
\hline ALT IU/L median (IQR) & $39(21-73)$ & $38(14-76)$ & $41(22-71.75)$ & 0.792 \\
\hline AST IU/L median (IQR) & $43(24-86.5)$ & $52(21-149)$ & $40(25.5-79.5)$ & 0.493 \\
\hline MCV IU/L median (IQR) & $96.9(91.2-100.5)$ & $97.8(91.8-101.7)$ & $96.8(91.2-99.8)$ & 0.522 \\
\hline Platelet count $\times 10^{9} / \mathrm{L}$ median $(\mathrm{IQR})$ & $206.5(129-271)$ & $203(101-303)$ & $206.5(133.3-262.5)$ & 0.645 \\
\hline Bilirubin $\mu \mathrm{mol} / \mathrm{L}$ median (IQR) & $10(4-16)$ & $10(4-20)$ & $10(4-15.5)$ & 0.624 \\
\hline FIB4 median (IQR) & $2.00(0.94-3.61)$ & $2.04(1.05-7.6)$ & $1.96(0.88-3.38)$ & 0.302 \\
\hline APRI median (IQR) & $0.64(0.3-2.08)$ & $0.64(0.28-2.71)$ & $0.63(0.3-1.9)$ & 0.552 \\
\hline AST:ALT ratio median (IQR) & $1.3(0.87-1.71)$ & $1.5(1.0-2.16)$ & $1.26(0.8-1.6)$ & 0.074 \\
\hline HA median (IQR) & $72.1(35.1-144.5)$ & & & \\
\hline PIIINP median (IQR) & $8.18(5.77-12.94)$ & & & \\
\hline TIMP1 median (IQR) & $265.7(198.6-364)$ & & & \\
\hline ELF median (IQR) & $9.62(8.93-10.6)$ & & & \\
\hline ELF range (lowest to highest) & $(6.87-13.78)$ & & & \\
\hline
\end{tabular}

$\mathrm{BMI}=$ Body Mass Index $\mathrm{U} / \mathrm{w}=$ units per week, $\mathrm{CLD}=$ Chronic Liver Disease, $\mathrm{T} 2 \mathrm{DM}=$ Type 2 Diabetes Mellitus, $\mathrm{ALT}=$ Alanine aminotranferase, $\mathrm{AST}=\mathrm{Aspartate}$ aminotransferase, MCV = Mean Corpuscular Volume, APRI = AST to platelet ratio index, HA = Hyaluronic acid, PIIINP=Procollagen $3 \mathrm{~N}$-terminal Peptide, $E L F=$ Enhanced Liver Fibrosis score

a At time of presentation to hospital or alcohol clinic

b Abnormal LFTs defined as raised transaminases or ALP + GGT (Not including isolated hyperbilirubinaemia (Gilbert's))

\section{Results of non-invasive fibrosis tests and LFTs}

LFTs were performed in $95 / 99$ patients of which $66.3 \%$ of these patients had abnormal LFTs (raised transaminases or ALP + GGT), with median ALT of 39 (21-73) and AST of 43 (24-86.5) (see Table 1). The median ELF score in the whole cohort was 9.62 (IQR 8.93-10.6, range 6.87-13.78). The ELF scores did not differ significantly between men and women $(p=0.435)$.

Twenty-eight participants (28.3\%) had an ELF score of $\geq 10.5$, indicating advanced fibrosis (Fig. 2). Of the 28 patients with advanced fibrosis (ELF $\geq 10.5), 8$ (28.6\%) had normal LFTs.

\section{Risk factors for high ELF score}

Clinical characteristics and baseline LFTs were first compared between patients without and with advanced fibrosis on the basis of ELF score $(<10.5 \mathrm{v} \geq 10.5)$. In addition, correlations were investigated between these same characteristics and ELF as a continuous variable. 

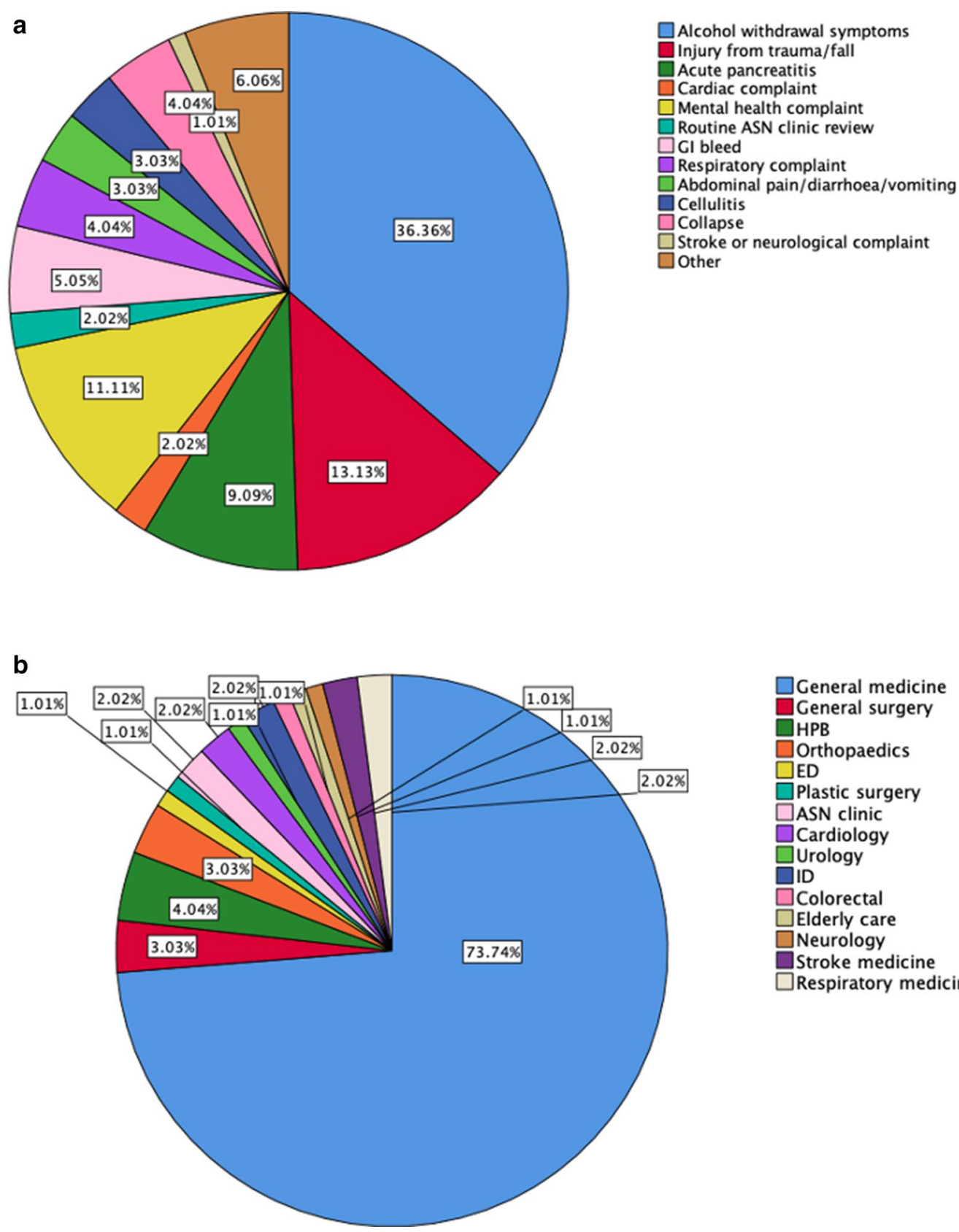

$\square$ General medicine
General surgery
$\square_{\text {HPB }}$
$\square$ Orthopaedics
$\square$ ED
$\square$ Plastic surgery
$\square$ ASN clinic
$\square$ Cardiology
$\square$ Urology
$\square$ ID
$\square$ Colorectal
$\square$ Elderly care
$\square$ Neurology
$\square$ Stroke medicine
$\square$ Respiratory medicine

Fig. 1 a Pie chart of reasons for presentation to hospital. b Pie chart of hospital specialty team (AUD=Alcohol Use Disorder, ASN=Alcohol Specialist Nurse, GI = Gastro-Intestinal, HPB = Hepato-Pancreato-Biliary, ED = Emergency Department, ID = Infectious Diseases)

\section{Comparison of clinical characteristics between patients with and without advanced fibrosis $(E L F \geq 10.5)$}

When comparing clinical characteristics with ELF score $<10.5$ versus $\geq 10.5$ there was no significant difference in age, sex, or BMI between the two groups (Table 1, Fig. 3d). There was also no significant difference in transaminase results, FIB4, or APRI scores between groups. Clinical signs of CLD were only found in patients with ELF score $\geq 10.5(\mathrm{n}=4)$. Patients in the advanced fibrosis group $(\geq 10.5)$ drank less alcohol than those with lower ELF scores (mean $112 \mathrm{U} / \mathrm{w}$, compared with $150 \mathrm{U} / \mathrm{w}, p=0.031$, Fig. 3b). However, there was no correlation observed between alcohol consumption and ELF score viewed as a continuous variable (Fig. 3a). Furthermore, multivariable regression analysis revealed no association between alcohol consumption 


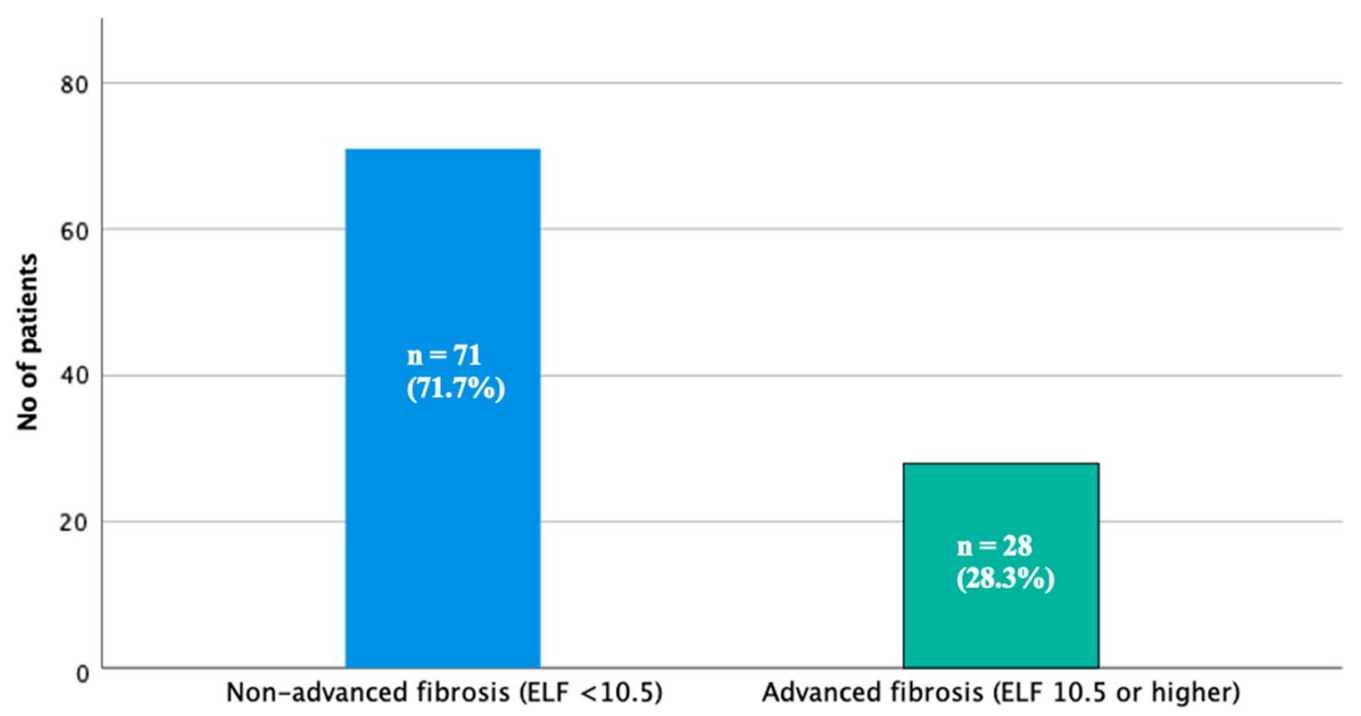

Fig. 2 Proportion of patients in study cohort with advanced fibrosis as assessed by ELF $\geq 10.5$
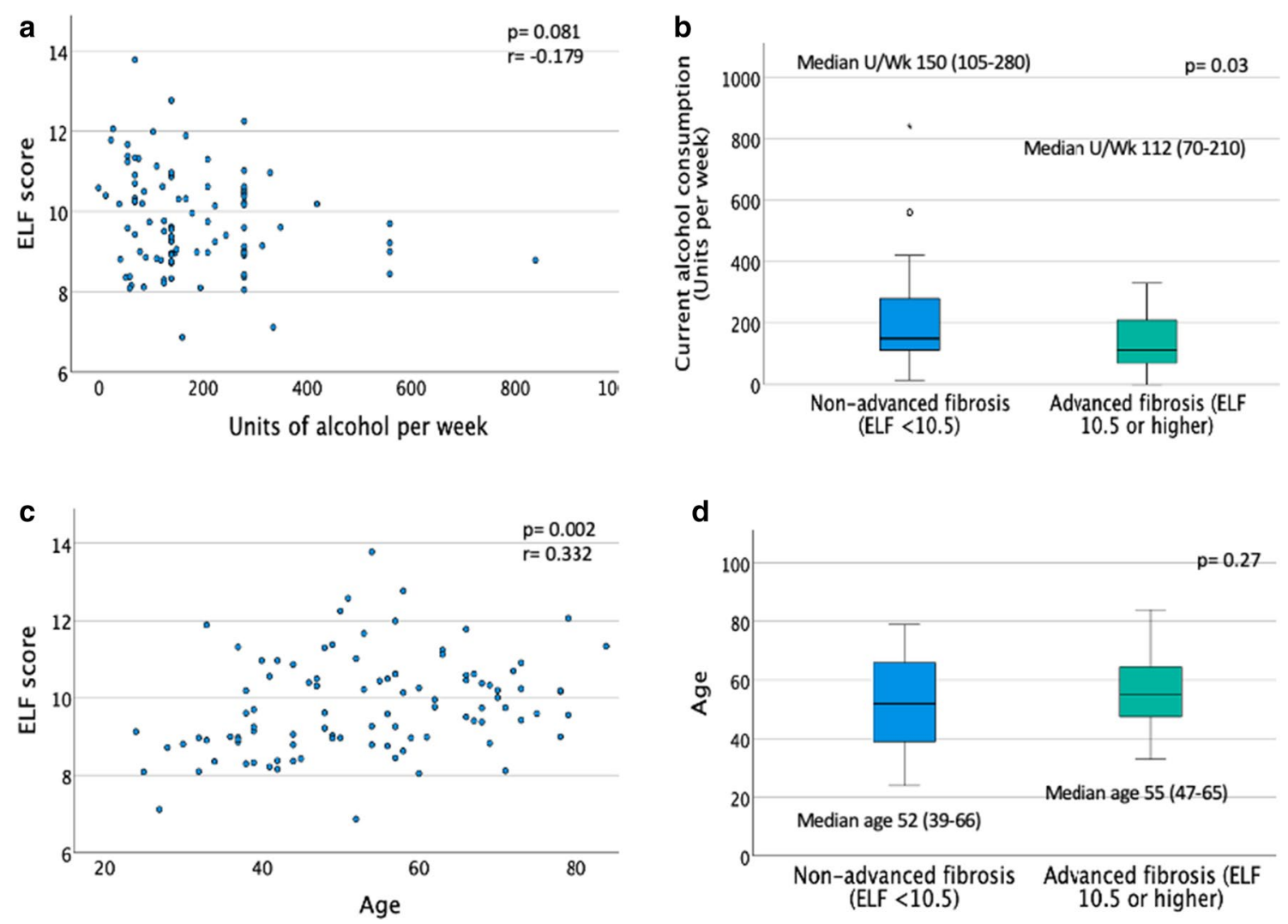

Fig. 3 Influence of alcohol and age on binary and continuous ELF scores. A: Scatter plot of ELF by alcohol units per week (Spearman Rho correlation, with $p$ value significance set at $0.05, r=$ correlation coefficient). B: Boxplot of alcohol consumption (Units per week) by presence or absence of advanced fibrosis (ELF $\geq 10.5$ ). Statistical test: Mann Whitney U, $p$ value significance set at 0.05 , median units per week displayed with IQR (interquartile range). C: Scatter plot of ELF by age (Spearman Rho correlation, with $p$ value significance set at $0.05, r=$ correlation coefficient). $D$ : Boxplot of age by presence or absence of advanced fibrosis ( $E L F \geq 10.5$ ). Statistical test: Mann Whitney $U, p$ value significance set at 0.05 , median age displayed with IQR (interquartile range) 
and ELF score (Table 2). There was no difference in the reported duration of alcohol excess in patients with ELF $<10.5$ compared to patients with ELF $>10.5$ (15 years (10-27.5 years) compared to 20 years (10-28 years); $p=0.357$ ).

Out of the three indirect biomarkers of fibrosis investigated (FIB4, APRI and AST:ALT ratio), AST:ALT ratios trended towards being higher in the advanced fibrosis group (median 1.5, IQR 1.0-2.16), than in the group with lower ELF scores (median 1.26, IQR 0.8-1.6; $p=0.074$ ). On univariate analysis the AST:ALT ratio did significantly predict advanced fibrosis based on ELF (OR 2.081 (95\% CI 1.145-3.779), $p=0.016$ (Table 2). On multivariable regression analysis, increasing AST:ALT ratio was the only variable significantly associated with ELF scores indicative of advanced fibrosis, when adjusted for age, alcohol intake, bilirubin, MCV, and ALP (OR 1.984, 95\%CI (1.014-3.884), $p=0.046$ (see Table 2).

\section{Factors associated with increasing ELF score}

When literature derived risk factors for liver fibrosis were analysed against a continuous ELF score, there was no longer a significant association between the amount of alcohol consumption (U/w) and ELF score $(p=0.081)$ (Fig. 3a) and this was confirmed in multivariable regression analysis, both using continuous ELF (Table 3) and binary ELF scores $</ \geq 10.5$ (Table 2). Alcohol intake was also analysed by grouping the units consumed per week into quartiles $(0-79 \mathrm{U} / \mathrm{w}, 80-140$ $\mathrm{U} / \mathrm{w}, 141-280 \mathrm{U} / \mathrm{w}$ and $281+\mathrm{U} / \mathrm{w}$. There was no significant difference in ELF score between the quartiles either when ELF was analysed as a continuous score or using the 10.5 threshold. (See Additional file 1: Tables S1 and S2).

ELF scores increased with increasing age (patients' total age range 24-84) on univariate analysis (Fig. 3c), $(r=0.303, p=0.002)$, and this was confirmed in multivariable analysis, when adjusted for alcohol intake, AST:ALT ratio, ALP, MCV and bilirubin ( $p=0.013$, 95\% CI $0.005-$ 0.042 ) (Table 3). For every 10-years increase in age, the ELF score increased by 0.24 .

ALT or AST were not associated with ELF score (either binary ELF of $>$ or $<10.5$, or continuous ELF (Fig. 4a-d and Table 2). Whilst AST:ALT ratio predicted advanced fibrosis when assessed using the 10.5 ELF threshold, a significant correlation was not seen between AST:ALT

Table 2 Factors associated with advanced fibrosis (ELF $\geq 10.5$ ), as determined by univariable and multivariable regression analyses

\begin{tabular}{|c|c|c|c|c|c|}
\hline Variable & $\begin{array}{l}\text { B (unstandardized } \\
\text { regression coefficient) }\end{array}$ & Univariable OR (95\% Cl) & $p$ value & Multivariable OR (95\% Cl) & $p$ value \\
\hline $\mathrm{Age}^{\mathrm{a}}$ & 0.018 & $1.018(0.987-1.050)$ & 0.264 & $1.010(0.972-1.049)$ & 0.609 \\
\hline Sex (male) & 0.054 & $1.055(0.412-2.699)$ & 0.911 & & \\
\hline $\mathrm{BMI}$ & -0.007 & $0.993(0.891-1.107)$ & 0.900 & & \\
\hline Current alcohol intake (U/Wk) & -0.005 & $0.995(0.991-1.000)$ & 0.041 & $0.995(0.990-1.000)$ & 0.070 \\
\hline Duration of alcohol excess & 0.009 & $1.009(0.975-1.044)$ & 0.613 & & \\
\hline Deprivation score & 0.000 & $1(1-1)$ & 0.323 & & \\
\hline Smoking (non-smoker) & -0.631 & $0.532(0.170-1.667)$ & 0.279 & & \\
\hline Abnormal LFTs at referral ${ }^{d}$ & 0.182 & $1.20(0.455-3.162)$ & 0.712 & & \\
\hline ALP & 0.006 & $1.006(0.999-1.012)$ & 0.099 & $1.004(0.998-1.011)$ & 0.190 \\
\hline $\mathrm{ALT}$ & 0.002 & $1.002(0.995-1.010)$ & 0.574 & & \\
\hline $\mathrm{MCV}^{\mathrm{b}}$ & 0.005 & $1.005(0.950-1.063)$ & 0.856 & $0.971(0.908-1.039)$ & 0.399 \\
\hline Platelet count & 0.000 & $1.000(0.996-1.003)$ & 0.786 & & \\
\hline Bilirubin & 0.025 & $1.026(0.988-1.065)$ & 0.187 & $0.999(0.953-1.047)$ & 0.966 \\
\hline $\mathrm{AST}^{\mathrm{C}}$ & 0.003 & $1.003(0.998-1.008)$ & 0.215 & & \\
\hline $\mathrm{FIB}^{\mathrm{C}}$ & 0.076 & 1.079 (0.996-1.169) & 0.063 & & \\
\hline AST/ALT ratio & 0.733 & $2.081(1.145-3.779)$ & 0.016 & $1.984(1.014-3.884)$ & 0.046 \\
\hline $\mathrm{APRI}^{\mathrm{C}}$ & 0.082 & $1.085(0.997-1.182)$ & 0.059 & & \\
\hline
\end{tabular}

$\mathrm{BMI}=$ Body Mass Index $\mathrm{U} / \mathrm{W}=$ units per week, $\mathrm{CLD}=$ Chronic Liver Disease, $\mathrm{T} 2 \mathrm{DM}=$ Type 2 Diabetes Mellitus, ALT =Alanine aminotranferase, $\mathrm{AST}=\mathrm{Aspartate}$ aminotransferase, $\mathrm{MCV}=$ Mean Corpuscular Volume, $\mathrm{APRI}=\mathrm{AST}$ to platelet ratio index, $\mathrm{ALP}=$ Alkaline Phosphatase, $\mathrm{OR}=\mathrm{Odds} \mathrm{Ratio}$

a Although $p$ value for age was above 0.25 in univariable logistic regression, it was $<0.05$ in correlation analysis with continuous ELF score, and is of clinical importance to investigate-so was included in this multivariable model

b Although $p$ value for MCV was above 0.25 in univariable logistic regression, it was $<0.05$ in correlation analysis with continuous ELF score, and so was included in this multivariable model

c Left out of multivariable analysis as would be affected by multi-collinearity with AST:ALT ratio, which was more highly significant in the univariate analysis

d Abnormal LFTs defined as raised transaminases or ALP + GGT. (Not including isolated hyperbilirubinaemia (Gilbert's)) 
Table 3 Summary of multiple regression analysis of factors associated with continuous ELF score

\begin{tabular}{|c|c|c|c|c|c|c|}
\hline \multirow[t]{2}{*}{ Model } & \multirow{2}{*}{$\begin{array}{l}\text { Unstandardized } \\
\text { coefficients } \\
\text { B }\end{array}$} & \multirow[t]{2}{*}{ Std. error } & \multirow{2}{*}{$\begin{array}{l}\text { Standardized } \\
\text { coefficients } \\
\text { Beta }\end{array}$} & \multirow[t]{2}{*}{ Sig } & \multicolumn{2}{|c|}{ 95.0\% Confidence interval for B } \\
\hline & & & & & Lower bound & Upper bound \\
\hline (Constant) & 6.701 & 1.580 & & 0.000 & 3.554 & 9.849 \\
\hline Age & 0.024 & 0.009 & 0.278 & 0.013 & 0.005 & 0.042 \\
\hline $\begin{array}{l}\text { Current alcohol intake } \\
(\mathrm{U} / \mathrm{wk})\end{array}$ & -0.001 & 0.001 & -0.105 & 0.334 & -0.003 & 0.001 \\
\hline ALP & 0.003 & 0.002 & 0.173 & 0.111 & -0.001 & 0.006 \\
\hline Bilirubin & 0.018 & 0.012 & 0.170 & 0.146 & -0.006 & 0.042 \\
\hline MCV & 0.014 & 0.016 & 0.094 & 0.374 & -0.018 & 0.046 \\
\hline AST:ALT ratio & 0.115 & 0.170 & 0.077 & 0.502 & -0.224 & 0.454 \\
\hline
\end{tabular}

(Dependent Variable: ELF score)

$(\mathrm{ALP}=$ Alkaline Phosphatase, $\mathrm{MCV}=$ Mean Corpuscular Volume, $\mathrm{AST}=$ Aspartate Aminotransferase, $\mathrm{ALT}=$ Alanine Aminotransferase $)$
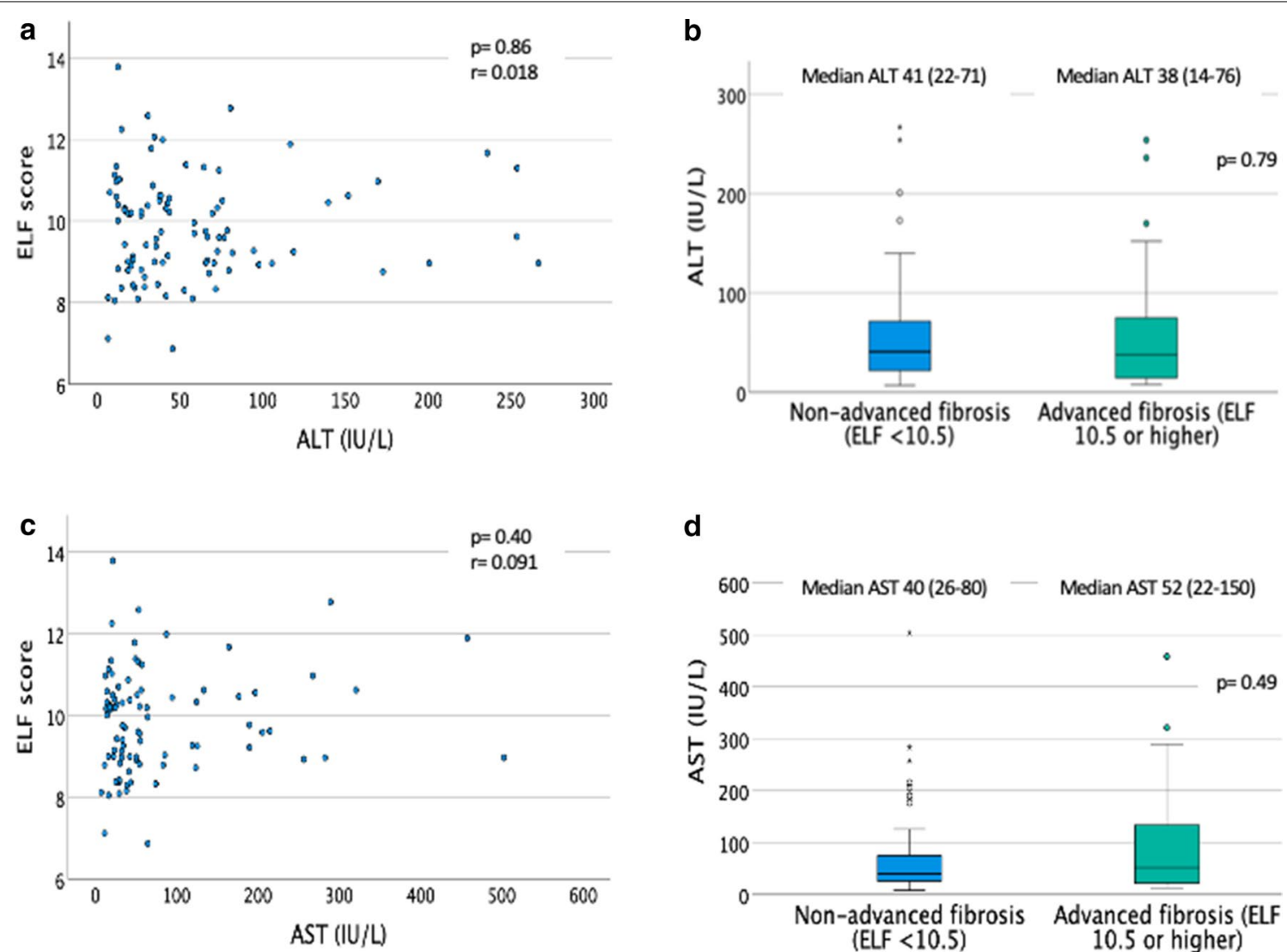

Fig. 4 Influence of ALT and AST on binary and continuous ELF scores. a Scatter plot of ELF by ALT value (Spearman Rho correlation, with $p$ value significance set at $0.05, r=$ correlation coefficient). $\mathbf{b}$ Boxplot of ALT by presence or absence of advanced fibrosis (ELF $\geq 10.5$ ). Statistical test: Mann Whitney U, $p$ value significance set at 0.05 , ALT displayed with IQR (interquartile range). c Scatter plot of ELF by AST value (Spearman Rho correlation, with $p$ value significance set at $0.05, r=$ correlation coefficient). $\mathbf{d}$ Boxplot of AST by presence or absence of advanced fibrosis (ELF $\geq 10.5)$. Statistical test: Mann Whitney U, $p$ value significance set at 0.05 , AST displayed with IQR (interquartile range) 
and continuous ELF score $(\mathrm{r}=0.12, p=0.27)$ (Additional file 1: Fig. S1a, b).

\section{FibroScan results}

Of the 28 patients with $E L F \geq 10.5$ who were offered a FibroScan appointment, only 18 attended, one of whom did not have a valid FibroScan reading, leaving 17 valid results (failure rate of $6 \%$ ). The mean FibroScan value was $10.9 \mathrm{kPa}( \pm 7.1 \mathrm{kpa})$, (total range of $4.2-25.3 \mathrm{kPa})$. Using a literature-derived threshold of $9.5 \mathrm{kPa}$ for advanced fibrosis [15], 10/17 (58.8\%) with ELF $\geq 10.5$ had a FibroScan value $<9.5 \mathrm{kPa}$.

\section{Discussion}

Over a quarter (28.3\%) of patients with AUD presenting to hospital for a variety of reasons had an ELF score of $\geq 10.5$, indicating the presence of advanced liver fibrosis. None of these patients had been assessed previously for liver fibrosis or referred to a liver specialist. All of them were at high risk of liver damage, with a current median alcohol consumption of $140 \mathrm{U} / \mathrm{w}$, and history of excess alcohol consumption lasting more than 15 years, and yet none had been investigated for liver disease during their index presentation or at any time previously. Moreover, $76 \%$ of the cohort had presented to hospital on a median of four times per person over the preceding five years without a diagnosis of ArLD, indicating missed opportunities for detection and treatment of liver fibrosis in a high-risk population. Missed opportunities for recognising and assessing liver damage in primary care were not investigated in this study, but none of the patients in this study had been referred to hepatology services for assessment of liver disease prior to diagnosis in this study.

We found that LFTs were not a reliable predictor of advanced fibrosis, with $28 \%$ of patients who had ELF $\geq 10.5$ having normal LFTs, in concordance with previous reports [7].

Whilst ELF scores were positively correlated with increasing age $(r=0.303, p=0.002)$, there was no difference in the median ages of those with or without advanced fibrosis, as determined by ELF scores $\geq 10.5$ or $<10.5$ respectively. ELF score has been found to correlate with age in some $[16,17]$, but not all studies [18] and it is unclear how much of the reported correlation is due to the increased likelihood of advanced fibrosis being present in older patients [19]. McPherson et al. [20] studied the impact of age on the performance of a range of NIT (NFS, FIB4, AST:ALT ratio, but not ELF) in detecting advanced fibrosis (compared with biopsy) in patients with Non-Alcoholic-Fatty-Liver-Disease (NAFLD), and found that all the tests performed less well in people over the age of 65 (with an increase in false positive rates in this age group). They suggested the use of adjusted thresholds for diagnosing advanced fibrosis in this age range. Fagan et al. [16] found increased risk of false positives with ELF above the age of 45, concluding that caution needs to be taken in interpreting ELF scores in older age groups. Thiele et al. [14] also reported increased false positive ELF results in people over 60, and advised caution in interpreting ELF in the over $60 \mathrm{~s}$. In contrast Parkes et al. [18] found no influence of age on ELF score in a cohort of patients with chronic hepatitis $\mathrm{C}(\mathrm{CHC})$ raising the possibility that age influences ELF score in NAFLD and ArLD but not in CHC.

The amount of alcohol consumed in U/w or duration of heavy drinking was not associated with ELF score in our cohort, and this was also the case in a large biopsy-controlled study of ELF in AUD by Thiele et al.[14] The same study also found that ALP was associated with increased ELF score, as observed in univariate analysis in this study, although not when adjusting for other factors in multivariable analysis.

Increasing AST:ALT ratio was the only other marker significantly associated with advanced fibrosis (ELF $\geq 10.5$ ) in this study (OR 1.984, 95\%CI 1.014-3.884, $p=0.046)$. Thiele et al. [14] found that AST:ALT had a Negative Predictive Value of $91 \%$ in a large biopsy-paired study and it may be that AST:ALT ratio could be used as a simple direct fibrosis test in addition to ELF in the assessment of advanced liver fibrosis in ArLD in a manner analogous to the combination of FIB4 and ELF in NAFLD [21] but this would require validation.

Whilst it has previously been reported that ELF scores may be influenced by inflammation $[17,22]$, we did not find any correlation between ELF score and ALT or AST, as markers of hepatic inflammation in this study, suggesting ELF was not influenced by inflammation in our cohort in keeping with findings of Thiele et al. study [14]. It must be noted, however, that patients with acute alcoholic hepatitis or acute liver injury from non-alcoholrelated causes were excluded as ELF is not validated in these settings.

Limitations of this study include the lack of paired biopsies that would have provided a more robust reference standard assessment of liver fibrosis. However the use of non-invasive tests to assess liver fibrosis in in this study is representative of current clinical practice within the NHS and in many other countries, where patients presenting to hospital with AUD are not routinely biopsied, partly due to increasing recognition of the imperfections of biopsy as a test for liver fibrosis due to sampling error, inter and intra observer variability and the costs and hazards associated with biopsy $[23,24]$. FibroScan was offered to all participants with ELF scores $\geq 10.5$, but only $18 / 28$ attended, of which 
valid readings were obtained for 17/18. Whilst FibroScan and ELF were discordant in 10/17 cases, FibroScan cannot be considered a robust reference standard measurement of fibrosis in ArLD, due to the impact of inflammation and alcohol on the accuracy of elastography. The small number of patients attending for FibroScan means that it is not possible to draw robust conclusions about the performance of FibroScan in this cohort. Furthermore, the poor attendance rate illustrates both the need to assess patients while they are inpatients, and the greater reliability of using a blood test to assess fibrosis that can be incorporated in routine investigations.

In common with routine practice, we relied on patients' self-reported alcohol intake extracted from clinical records, an approach that is likely to be inaccurate. Unfortunately, it is not local routine practice to obtain AUDIT scores but these would provide additional valuable information about drinking behaviour. Fibrosis was assessed using a single ELF test at the start of the patients' hospital admission. Although liver stiffness as measured by FibroScan reduces significantly on withdrawal of alcohol [25-27], a study of ten patients found that there was no significant difference in the ELF scores recorded from intoxicated patients when re-tested two weeks after alcohol withdrawal [28] but the impact of drinking on ELF score needs further investigation.

Overall, this study has highlighted the missed opportunities for detecting liver fibrosis in at-risk patients in a hospital setting. Alcohol use disorder must be viewed as a multimorbid condition with psycho-social morbidity and the potential to damage every organ in the body. However, alcohol related liver disease accounts for much of the mortality and costs of drinking and accurate and relatively inexpensive blood tests are now available that permit detection of liver damage in all those at risk. It could be argued that there is no longer any excuse to miss the diagnosis of liver fibrosis in patients presenting to hospital with AUD. Whilst people with AUD encompass some of the more socially disadvantaged members of society that may find engaging with routine health services difficult, it is imperative that all opportunities to detect liver fibrosis should be taken especially on those occasions when they present to hospital with complications of AUD or other conditions.

BSG guidance now recommends non-invasive fibrosis testing for people with high-risk alcohol intake $(>35 \mathrm{U} / \mathrm{w}$ in women, $>50 \mathrm{U} / \mathrm{w}$ in men) [7] with either FibroScan or ELF. This study emphasises the importance of implementing this guidance and incorporating it into hospital guidelines in emergency departments and in alcohol care teams [29] to improve the detection of advanced fibrosis in people with AUD.

\section{Abbreviations}

ArLD: Alcohol related liver disease; CLD: Chronic liver disease; AUD: Alcohol use disorder; NIT: Non-invasive test; ELF: Enhanced liver fibrosis; ALT: Alanine aminotransferase; AST: Aspartate aminotransferase; ALP: Alkaline phosphatase; FIB4: Fibrosis 4 score; APRI: AST to platelet ratio index; BMI: Body mass index; T2DM: Type II diabetes mellitus; OR: Odds ratio; Cl: Confidence interval; U/w: Units per week; BSG: British Society of Gastroenterology.

\section{Supplementary Information}

The online version contains supplementary material available at https://doi. org/10.1186/s12876-021-01728-2.

Additional file 1. Table S1: Multiple logistic regression analysis to investigate for effect of alcohol unit quartiles on presence or absence of advanced fibrosis (as per ELF $\geq 10.5$ ). Table S2: Multiple linear regression analysis to investigate for effect of alcohol unit quartiles on continuous ELF score. Fig. S1: Influence of AST:ALT ratio on binary and continuous ELF scores. a Scatter plot of ELF by AST:ALT ratio (Spearman Rho correlation, with $p$ value significance set at $0.05, r=$ correlation coefficient). $\mathbf{b}$ Boxplot of AST:ALT ratio by presence or absence of advanced fibrosis (ELF $\geq 10.5$ ). Statistical test: Mann Whitney U, $p$ value significance set at 0.05, AST:ALT ratio displayed with IQR (interquartile range).

\section{Acknowledgements}

We would like to acknowledge the expert input from the alcohol specialist nurse, Mr. Kevin Raeburn, to whom all the patients in this study were referred for review.

\section{Authors' contributions}

FR set up the study, collected the data, performed the data analysis and wrote the first draft of the manuscript. SC contributed to the collection of data and the editing of the manuscript. JP-G contributed to the statistical analysis. WR conceived the study, oversaw the study and reviewed and edited the manuscript drafts. RW, ST and AR contributed to the analysis methodology, and the review and editing of the manuscript. All authors approved the final version of the paper. The corresponding author attests that all listed authors meet authorship criteria and that no others meeting the criteria have been omitted. All authors read and approved the final manuscript.

\section{Funding}

This study was supported by funding from WMR's National Institute for Health Research Senior Investigator Award (Award No. 200249). WMR is an NIHR Senior Investigator and is supported by the NIHR University College London Hospitals Biomedical Research Centre. JPG was supported by the United Kingdom National Institute for Health Research (NIHR) Applied Research Collaboration North Thame (ARC North Thames) at Bart's Health NHS Trust.

Availability of data and materials

On publication of this article, the dataset will be made available from the corresponding author on reasonable request.

\section{Declarations}

\section{Ethics approval and consent to participate}

This study was reviewed by the Royal Free London NHS Foundation Trust Research and Development Office and deemed to be a service evaluation of an established pathway of care, using an established and CE marked diagnostic test. Using the Health Research Authority Tool (http://www.hra-decisionto ols.org.uk/research/question 1.html) the study did not meet the criteria that would require it to undergo external ethical review but rather to be registered as a service evaluation conducted within the NHS Trust. It was registered with the Royal Free London Audit and Service Evaluation Department on the $10^{\text {th }}$ October 2018. Based on this review of the study the Royal Free London NHS Foundation Trust Research and Development Office deemed that there was no necessity to obtain informed consent from participants in this service evaluation. Although not deemed to be "research" using the HRA Tool, the study was in compliance with the regulatory requirements for service 
evaluations and was conducted in accordance with the principles of the WMA Declaration of Helsinki.

\section{Consent for publication}

Not applicable.

\section{Competing interests}

WMR is an inventor of the ELF test but receives no related royalties. WMR has received speaker's fees from Siemens Healthineers. The other authors declare no competing interests.

\section{Author details}

${ }^{1}$ Institute for Liver and Digestive Health, Division of Medicine, University College London, Royal Free Campus, Rowland Hill Street, Hampstead, London NW3 2PF, UK. ${ }^{2}$ First Department of Internal Medicine, San Matteo Hospital Foundation, University of Pavia, Pavia, Italy. ${ }^{3}$ Department of Applied Health Research, University College London, London, UK. ${ }^{4}$ Institute for Global Health, University College London, London, UK. ${ }^{5}$ Barts Health NHS Trust, London, UK. ${ }^{6}$ Institute for Liver and Digestive Health, UCL Division of Medicine, Royal Free Campus, London NW3 2QG, UK.

\section{Received: 27 October 2020 Accepted: 18 March 2021}

Published online: 31 March 2021

\section{References}

1. British-Liver-Trust. Alcohol Related Liver Disease: Facts about Alcohol Related Liver Disease 2019. https://britishlivertrust.org.uk/informationand-support/living-with-a-liver-condition/liver-conditions/alcohol/. Accessed 20th Nov 2020.

2. Williams R, Aspinall R, Bellis M, Camps-Walsh G, Cramp M, Dhawan A, et al. Addressing liver disease in the UK: a blueprint for attaining excellence in health care and reducing premature mortality from lifestyle issues of excess consumption of alcohol, obesity, and viral hepatitis. Lancet. 2014;384(9958):1953-97.

3. NHS-Digital. Statistics on Alcohol, England 2020. https://digital.nhs.uk/ data-and-information/publications/statistical/statistics-on-alcohol/2020. Accessed 20th Nov 2020.

4. Rice P, Drummond C. The price of a drink: the potential of alcohol minimum unit pricing as a public health measure in the UK. Br J Psychiatry. 2012;201(3):169-71.

5. Sheron N, Moore M, O'Brien W, Harris S, Roderick P. Feasibility of detection and intervention for alcohol-related liver disease in the community: the Alcohol and Liver Disease Detection study (ALDDeS). Br J Gen Pract. 2013;63(615):e698-705.

6. Hussain A, Patel PJ, Rhodes F, Srivastava A, Patch D, Rosenberg W. Decompensated cirrhosis is the commonest presentation for NAFLD patients undergoing liver transplant assessment. Clin Med (Lond). 2020:20(3):313-8.

7. Newsome PN, Cramb R, Davison SM, Dillon JF, Foulerton M, Godfrey EM, et al. Guidelines on the management of abnormal liver blood tests. Gut. 2018;67(1):6-19.

8. Lo GH, Lai KH, Cheng JS, Lin CK, Hsu PI, Chiang HT. Prophylactic banding ligation of high-risk esophageal varices in patients with cirrhosis: a prospective, randomized trial. J Hepatol. 1999;31(3):451-6.

9. Sarin SK, Guptan RK, Jain AK, Sundaram KR. A randomized controlled trial of endoscopic variceal band ligation for primary prophylaxis of variceal bleeding. Eur J Gastroenterol Hepatol. 1996;8(4):337-42.

10. Terg R, Fassio E, Guevara M, Cartier M, Longo C, Lucero R, et al. Ciprofloxacin in primary prophylaxis of spontaneous bacterial peritonitis: a randomized, placebo-controlled study. J Hepatol. 2008;48(5):774-9.

11. Trinchet JC, Chaffaut C, Bourcier V, Degos F, Henrion J, Fontaine $H$, et al. Ultrasonographic surveillance of hepatocellular carcinoma in cirrhosis: a randomized trial comparing 3- and 6-month periodicities. Hepatology. 2011;54(6):1987-97.

12. Santi V, Trevisan F, Gramenzi A, Grignaschi A, Mirici-Cappa F, Del Poggio $P$, et al. Semianual surveillance is superior to annual surveillance for the detection of early hepatocellular carcinoma and patient survival. J Hepatol. 2010;53(2):291-7.

13. Harrison P, Hogan BJ, Floros L, Davies E, Guideline-Development-Group. Assessment and management of cirrhosis in people older than 16 years: summary of NICE guidance. BMJ. 2016;354:i2850.

14. Thiele M, Madsen BS, Hansen JF, Detlefsen S, Antonsen S, Krag A. Accuracy of the enhanced liver fibrosis test vs. fibrotest, elastography, and indirect markers in detection of advanced fibrosis in patients with alcoholic liver disease. Gastroenterology. 2018;154(5):1369-79.

15. Pavlov CS, Casazza G, Nikolova D, Tsochatzis E, Gluud C. Systematic review with meta-analysis: diagnostic accuracy of transient elastography for staging of fibrosis in people with alcoholic liver disease. Aliment Pharmacol Ther. 2016;43(5):575-85.

16. Fagan KJ, Pretorius CJ, Horsfall LU, Irvine KM, Wilgen U, Choi K, et al. ELF score $\geq 9.8$ indicates advanced hepatic fibrosis and is influenced by age, steatosis and histological activity. Liver Int. 2015;35(6):1673-81.

17. Lichtinghagen R, Pietsch D, Bantel H, Manns MP, Brand K, Bahr MJ. The enhanced liver fibrosis (ELF) score: normal values, influence factors and proposed cut-off values. J Hepatol. 2013;59(2):236-42.

18. Parkes J, Guha IN, Roderick P, Harris S, Cross R, Manos MM, et al. Enhanced liver fibrosis (ELF) test accurately identifies liver fibrosis in patients with chronic hepatitis C. J Viral Hepat. 2011;18(1):23-31.

19. Poynard T, Ratziu V, Charlotte F, Goodman Z, McHutchison J, Albrecht J. Rates and risk factors of liver fibrosis progression in patients with chronic hepatitis c. J Hepatol. 2001;34(5):730-9.

20. McPherson S, Hardy T, Dufour JF, Petta S, Romero-Gomez M, Allison M, et al. Age as a confounding factor for the accurate non-invasive diagnosis of advanced NAFLD fibrosis. Am J Gastroenterol. 2017;112(5):740-51.

21. Srivastava A, Gailer R, Tanwar S, Trembling P, Parkes J, Rodger A, et al. Prospective evaluation of a primary care referral pathway for patients with non-alcoholic fatty liver disease. J Hepatol. 2019;71(2):371-8.

22. Wahl K, Rosenberg W, Vaske B, Manns MP, Schulze-Osthoff K, Bahr MJ, et al. Biopsy-controlled liver fibrosis staging using the enhanced liver fibrosis (ELF) score compared to transient elastography. PLoS ONE. 2012;7(12).

23. Maharaj B, Maharaj RJ, Leary WP, Cooppan RM, Naran AD, Pirie D, et al. Sampling variability and its influence on the diagnostic yield of percutaneous needle biopsy of the liver. Lancet. 1986;1(8480):523-5.

24. Regev A, Berho M, Jeffers LJ, Milikowski C, Molina EG, Pyrsopoulos NT, et al. Sampling error and intraobserver variation in liver biopsy in patients with chronic HCV infection. Am J Gastroenterol. 2002;97(10):2614-8.

25. Trabut JB, Thepot V, Nalpas B, Lavielle B, Cosconea S, Corouge M, et al. Rapid decline of liver stiffness following alcohol withdrawal in heavy drinkers. Alcohol Clin Exp Res. 2012;36(8):1407-11.

26. Bardou-Jacquet E, Legros L, Soro D, Latournerie M, Guillygomarc'h A, Le Lan $C$, et al. Effect of alcohol consumption on liver stiffness measured by transient elastography. World J Gastroenterol. 2013;19(4):516-22.

27. Gelsi E, Dainese R, Truchi R, Marine-Barjoan E, Anty R, Autuori M, et al. Effect of detoxification on liver stiffness assessed by Fibroscan(R) in alcoholic patients. Alcohol Clin Exp Res. 2011;35(3):566-70.

28. Ponomarenko Y, Leo MA, Kroll W, Lieber CS. Effects of alcohol consumption on eight circulating markers of liver fibrosis. Alcohol Alcohol. 2002;37(3):252-5.

29. Chapman R, Middleton J. The NHS long term plan and public health. BMJ. 2019;364:1218.

\section{Publisher's Note}

Springer Nature remains neutral with regard to jurisdictional claims in published maps and institutional affiliations. 